\title{
Pathologies vasculaires intestinales
}

\section{O. Corcos}

C) Springer-Verlag France 2010

Le spectre des pathologies vasculaires intestinales est large, allant de la simple colite ischémique à l'infarctus mésentérique massif souvent fatal. Les pathologies vasculaires peuvent être aiguës ou chroniques, évolutives ou indolentes, artérielles ou veineuses, occlusives ou non occlusives, emboliques ou athéromateuses, inflammatoires ou radiques. Lorsque la vascularisation artérielle est compromise de façon aiguë et en dessous du seuil tolérable par la paroi intestinale, dont témoigne le début de la douleur abdominale, un compte à rebours de 12 à 24 heures se met en route, nécessitant une intervention thérapeutique en extrême urgence afin de sauver la vie du patient.

Parmi les traitements rapportés dans la littérature, aucun n'a donné lieu à un essai contrôlé. Les seules références bibliographiques existantes portent sur des séries rétrospectives, sur des avis d'experts, sur des recommandations ou bien sur des études non contrôlées de faibles effectifs. L'hétérogénéité des formes cliniques et étiologiques, la relative rareté de cette pathologie, l'absence de gold standard diagnostique disponible dans l'urgence ne permettent pas la réalisation d'études contrôlées, ce qui condamne trop souvent le clinicien à improviser une stratégie selon sa propre expérience.

Nous nous efforcerons pourtant dans ce dossier, à la lumière d'une littérature insuffisante mais abondante, de démembrer le spectre des pathologies vasculaires et de rapporter les principaux traitements ayant permis d'en améliorer le pronostic. Une stratégie relativement standardisée pourra ainsi être proposée afin d'éviter au chirurgien la funeste alternative entre voir le patient mourir du fait d'un trop grand retard diagnostique et la fameuse open/close procédure.

O. Corcos $(\bowtie)$

Service de Gastroentérologie, MICI,

Assistance-Nutritive et Transplantation Intestinale,

Pôles des Maladies de 1'Appareil Digestif,

Hôpital Beaujon,

100 , boulevard du Général-Leclerc,

F-92110 Clichy, France

e-mail : olivier.corcos@bjn.aphp.fr 